\title{
Genetic and clinical correlates of entosis in pancreatic ductal adenocarcinoma
}

\author{
Akimasa Hayashi $\mathbb{D}^{1,2} \cdot$ Aslihan Yavas $^{1,3} \cdot$ Caitlin A. Mclntyre ${ }^{1,2} \cdot$ Yu-jui Ho $^{4} \cdot$ Amanda Erakky $^{1} \cdot$ Winston Wong $^{5}$. \\ Anna M. Varghese ${ }^{5}$. Jerry P. Melchor ${ }^{1,2}$ - Michael Overholtzer ${ }^{6}$ - Eileen M. O'Reilly $\mathbb{B}^{1,5} \cdot$ David S. Klimstra ${ }^{1,3}$. \\ Olca Basturk $\mathbb{D}^{3} \cdot$ Christine A. lacobuzio-Donahue $\mathbb{I}^{1,2,3}$
}

Received: 26 February 2020 / Revised: 5 April 2020 / Accepted: 5 April 2020 / Published online: 29 April 2020

(c) The Author(s) 2020. This article is published with open access

\begin{abstract}
Entosis is a type of regulated cell death that promotes cancer cell competition. Though several studies have revealed the molecular mechanisms that govern entosis, the clinical and genetic correlates of entosis in human tumors is less well understood. Here we reviewed entotic cell-in-cell (CIC) patterns in a large single institution sequencing cohort (MSK IMPACT clinical sequencing cohort) of more than 1600 human pancreatic ductal adenocarcinoma (PDAC) samples to identify the genetic and clinical correlates of this cellular feature. After case selection, 516 conventional PDACs and 21 ASCs entered this study and 45,000 HPFs (median 80 HPFs per sample) were reviewed; 549 entoticCICs were detected through our cohort. We observed that entotic-CIC occurred more frequently in liver metastasis compared with primary in PDAC. Moreover, poorly differentiated adenocarcinoma or adenosquamous carcinoma had more entotic-CIC than well or moderately differentiated adenocarcinoma. With respect to genetic features TP53 mutations, KRAS amplification, and MYC amplification were significantly associated with entosis in PDAC tissues. From a clinical standpoint entotic CICs were independently associated with a poor prognosis by multivariate Cox regression analysis when considering all cases or primary PDACs specifically. These results provide a contextual basis for understanding entosis in PDAC, a highly aggressive cancer for which molecular insights are needed to improve survival.
\end{abstract}

\section{Introduction}

Entosis is a type of regulated cell death that originates from actomyosin-dependent cell-in-cell (CIC) internalization and

Supplementary information The online version of this article (https:// doi.org/10.1038/s41379-020-0549-5) contains supplementary material, which is available to authorized users.

Olca Basturk

BasturkO@mskcc.org

$\triangle$ Christine A. Iacobuzio-Donahue

iacobuzc@mskcc.org

1 The David M. Rubenstein Center for Pancreatic Cancer Research, Sloan Kettering Institute, Memorial Sloan Kettering Cancer Center, New York, NY, USA

2 Human Oncology and Pathogenesis Program, Sloan Kettering Institute, Memorial Sloan Kettering Cancer Center, New York, NY, USA is executed by lysosomes [1]. Entosis was first discovered as a nonapoptotic cell death process in 2007 [2], and thought to have an advantage for cancer cell survival by promoting cell competition through direct cell-cell interactions unlike other forms of cell death including ferroptosis that occurs at the level of individual cells [3]. Entosis is induced microenvironmentally by glucose starvation [4] and has been associated with TP53 mutations [5]. Moreover, activated $K R A S$ and Rac signaling have been implicated in

3 Department of Pathology, Memorial Sloan Kettering Cancer Center, New York, NY, USA

4 Cancer Biology and Genetics Program, Sloan Kettering Institute, Memorial Sloan Kettering Cancer Center, New York, NY, USA

5 Department of Medicine, Memorial Sloan Kettering Cancer Center, New York, NY, USA

6 Cell Biology Program, Sloan Kettering Institute, Memorial Sloan Kettering Cancer Center, New York, NY, USA 
a MSK IMPACT Clinical Sequencing Cohort

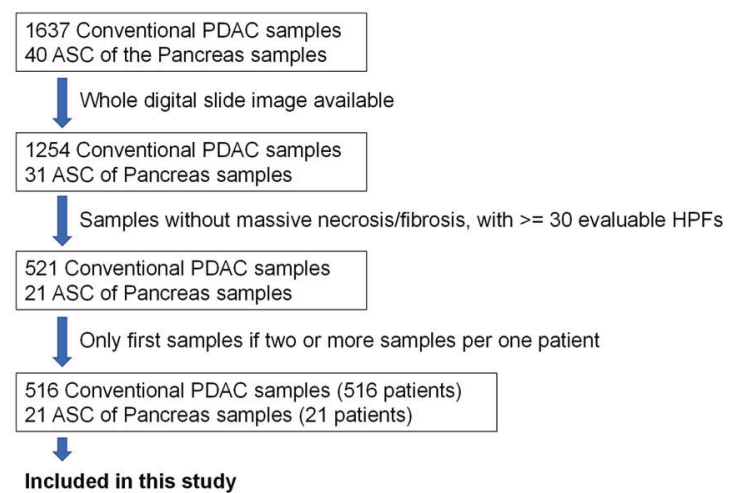

b

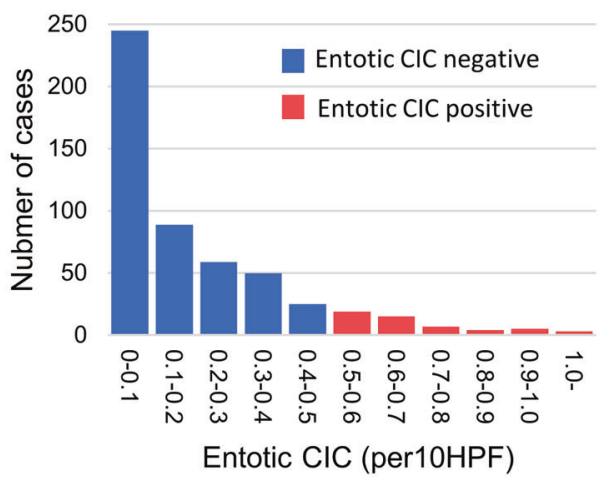

C
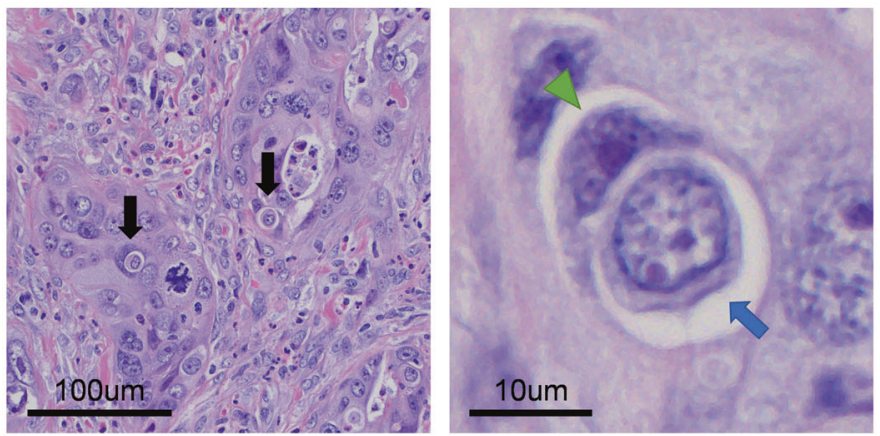

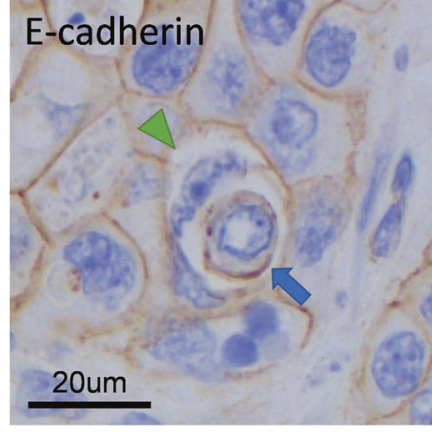

Fig. 1 Overview of study set and entotic-CICs. a Schematic of sample selection for current study. b Distribution of entotic-CIC (per 10 HPFs) in PDACs. c Representative histomorphologic and immunohistochemical (E-cadherin) images of entotic-CICs. Entotic-CICs with intervening vacuolar spaces (arrows) were confirmed in low power view. High power view in the middle illustrating a loser cell (blue arrow) engulfed by a winner cell (green arrowhead). E-cadherin confirms entotic-CICs due to the presence of membranous labeling of both the winner and loser cells. establishment of winner cell status and in promoting cell death [6].

While studies in vitro have revealed molecular mechanisms of entosis, translational studies exploring the clinicopathological and genetic correlates of entosis in patients are limited. The available data point to entosis as a correlate of aggressive behavior. In rectal cancer, head and neck squamous cell carcinoma (HNSCC) and in lung cancer, a CIC pattern has been associated with poor prognosis $[5,7,8]$. In breast cancer, the CIC pattern is more prevalent in high grade tumors [9]. In pancreatic ductal adenocarcinoma (PDAC), we have recently shown that mutant TP53 status and/or metabolic related gene expression changes are positively correlated with formation of entotic CIC [10]. However, the extent to which entosis correlates with metastatic propensity remains unknown, as does the extent to which genes other than TP53 contribute to this phenotypic change. For this reason, we investigated the CIC pattern in PDAC using a large single institution cohort of both primary and metastasis samples for which targeted sequencing data of more than 400 genes are available.

\section{Materials and methods}

\section{MSK IMPACT clinical sequencing cohort clinical and genetic information}

All genetic and clinical information and digital whole slide images were obtained from the MSK IMPACT Clinical Sequencing Cohort database through cBioPortal (version2.2.0) [11]. Genetic information available for each case was based on results of a targeted sequencing panel for up to 468 known and predicted cancer genes as previously described [12]. Treatment information (chemotherapy or chemoradiation therapy) was obtained from an IRB approved and HIPAA compliant RED Cap Database (version 1.0) available through the Center for Pancreatic Cancer Research.

\section{MSK IMPACT clinical sequencing cohort case selection}

Summary of case selection was shown in Fig. 1a. All genetic and clinical information and digital whole slide 
images were obtained from the MSK IMPACT Clinical Sequencing Cohort database through cBioPortal (version2.2.0) [11]. Of the PDAC cases, 1637 (conventional or tubular) ductal adenocarcinomas (PDAC) and 40 adenosquamous carcinomas (ASCs) for which both genetic information and digital images were available for study. All hematoxylin and eosin (H\&E) digital slides were reviewed by two gastrointestinal pathologists (AH and CAI-D). Samples with massive necrosis and/or degeneration and/or fibrosis, or small biopsy samples with $<30$ evaluable highpower fields (HPFs) were excluded from this study. In addition, in each sample, tumor regions with degeneration and/or necrosis and/or low tumor content $(<10 \%)$ were excluded. Finally, a sample set of 537 pancreatic neoplasms (516 conventional PDACs and 21 ASCs) (Fig. 1a) were included in this study.

\section{Histologic definition of entotic CIC}

Entotic CIC structures were defined using $\mathrm{H} \& \mathrm{E}$ images as reported previously [10] as originally proposed by MacKay [5]: cytoplasm of the host cell (winner or engulfing cell), nucleus of the host cell (typically crescent-shaped, binucleate, or multilobular and pushed against the cytoplasmic wall), an intervening vacuolar space surrounding the internalized cell (loser), cytoplasm of internalized cell, and nucleus of internalized cell (often round in shape and located centrally or acentrically). If internalized and/or engulfing cells were undergoing typical mitosis or suspected apoptotic changes they were excluded from analysis. Apoptotic changes were characterized by pyknotic nuclei, nuclear fragmentation, and loss of nuclear detail.

\section{MSK IMPACT clinical sequencing cohort slide review}

Digital whole slides available for the 537 cases selected for study from the MSK IMPACT Clinical Sequencing Cohort were visualized through Smart Slide Viewer (version 1.3.1583). All evaluable tumor regions submitted to the IMPACT sequencing in digital slides were reviewed by two pathologists (AH and CID). A total 45,000 HPFs (range 30-197 HPFs, median 80 HPFs per sample) were reviewed and all potential or suspected entotic-CICs $(\sim 1000$ unique images) were captured and reviewed with discussion. Entotic-CICs were quantified by calculating the average number per ten HPFs. Based on this information we divided the cohort into two groups as previously reported [10]. "Entotic-CIC positive" PDACs were defined as those with 0.5 or more entotic CICs per ten HPFs, corresponding to the top $\sim 10 \%$ in this study. PDACs not meeting these criteria were defined as "entotic-CIC negative". Tumor grading (well-, moderately-, poorly differentiated adenocarcinoma) of all cases was determined based on WHO classification [13].

\section{Immunohistochemistry of E-cadherin}

The histologic classification of entosis in 15 representative cases with entotic-CICs from the MSK IMPACT Clinical Sequencing Cohort was validated using immunohistochemistry for E-cadherin [14]. Five micrometer unstained sections were cut from the FFPE blocks of each case, and immunolabeled with a mouse monoclonal antibody against E-cadherin (BD Biosciences, Catalog No. 610181, clone 36/E-Cadherin, $5 \mathrm{ug} / \mathrm{ml}$ ) according to optimized protocol on a Ventana Benchmark XT autostainer (Ventana Medical Systems Inc.) in the MSK Pathology Core Lab. Appropriate positive and negative controls were included in each run.

\section{Genetic features of cohort}

Using cBioportal as a guide the top ten most frequently mutated genes and the top five most frequent copy number alterations in the cohort of 537 patients were included for correlation to entotic-CICs.

\section{Autopsy cohort}

Matched primary and metastatic tissues from three patients in the Last Wish Program at Memorial Sloan Kettering Cancer Center and 21 patients from the Gastrointestinal Cancer Rapid Medical Donation Program at Johns Hopkins Hospital were used for the validation autopsy cohort. All slides were reviewed by two gastrointestinal pathologists (AH and CID). At least two slides (median eight slides, range 2-12) and $50 \mathrm{HPFs}$ (median $379 \mathrm{HPFs}$, range 52-1078) for each primary and metastasis site were evaluated for entotic-CICs. Only metastases from the liver, lung, and/or peritoneal cavity were included.

\section{Ethics statement}

This study was approved by the Review Boards of Johns Hopkins School of Medicine and Memorial Sloan Kettering Cancer Center.

\section{Statistics}

All statistics and graphs were performed and made using GraphPad Prism (version 8.2.1) and/or XLSTAT (version 2019.4.2). Each analysis method was described in Results or Figure Legends. Statistical significance was considered if $P$ value is $<0.05$. 
Table 1 Clinicopathologic characteristics of entotic-CIC positive PDAC.

\begin{tabular}{|c|c|c|c|c|c|c|}
\hline \multirow[t]{2}{*}{ Factor } & & \multirow[b]{2}{*}{ Total } & \multicolumn{3}{|c|}{ Entotic-CIC } & \multirow[b]{2}{*}{$P$ Value } \\
\hline & & & Positive & Negative & $\%$ Positive & \\
\hline \multirow[t]{2}{*}{ Age } & $>65$ & 317 & 45 & 272 & $14.2 \%$ & \multirow[t]{2}{*}{0.364} \\
\hline & $\leq 65$ & 220 & 25 & 195 & $11.4 \%$ & \\
\hline \multirow[t]{2}{*}{ Gender $^{\mathrm{a}}$} & Male & 299 & 39 & 260 & $13.0 \%$ & \multirow[t]{2}{*}{1.000} \\
\hline & Female & 237 & 31 & 206 & $13.1 \%$ & \\
\hline \multirow[t]{2}{*}{ Histology $y^{b}$} & PDAC & 516 & 65 & 451 & $12.6 \%$ & \multirow[t]{2}{*}{0.175} \\
\hline & ASC & 21 & 5 & 16 & $23.8 \%$ & \\
\hline \multirow[t]{2}{*}{ Location } & Primary & 415 & 40 & 375 & $9.6 \%$ & \multirow[t]{2}{*}{$<0.0001$} \\
\hline & Metastasis & 122 & 30 & 92 & $24.6 \%$ & \\
\hline
\end{tabular}

The bold value means statistically significant $P$ value.

Each $P$ value was calculated with the Fisher's exact test, two-sided.

${ }^{\mathrm{a}}$ Gender information was not available for one patient.

${ }^{\mathrm{b}} P D A C$ Pancreatic ductal adenocarcinoma, ASC Adenosquamous carcinoma.

\begin{tabular}{|c|c|c|c|c|c|c|}
\hline \multirow[t]{2}{*}{ Factor } & & \multirow[b]{2}{*}{ Total } & \multicolumn{3}{|c|}{ Entotic-CIC } & \multirow[b]{2}{*}{$P$ Value } \\
\hline & & & Positive & Negative & $\%$ Positive & \\
\hline \multirow[t]{4}{*}{ Tumor grade } & Well & 75 & 2 & 73 & $2.7 \%$ & \multirow[t]{4}{*}{$<0.0001$} \\
\hline & Moderately & 210 & 10 & 200 & $4.8 \%$ & \\
\hline & Poorly & 111 & 23 & 88 & $20.7 \%$ & \\
\hline & $\mathrm{ASC}^{\#}$ & 19 & 5 & 14 & $26.3 \%$ & \\
\hline \multirow[t]{2}{*}{ Lymph node metastasis ${ }^{a}$} & Positive & 290 & 28 & 262 & $9.7 \%$ & \multirow[t]{2}{*}{1.000} \\
\hline & Negative & 118 & 11 & 107 & $9.3 \%$ & \\
\hline \multirow[t]{2}{*}{ Neoadjuvant therapy ${ }^{\mathrm{b}}$} & Yes & 69 & 3 & 66 & $4.3 \%$ & \multirow[t]{2}{*}{0.120} \\
\hline & No & 340 & 37 & 303 & $10.9 \%$ & \\
\hline
\end{tabular}

The bold value means statistically significant $P$ value.

Each $P$ value was calculated with the Fisher's exact test, two-sided.

${ }^{a}$ Lymph node metastasis information is available for 408 surgical resected cases.

${ }^{b}$ Neoadjuvant therapy information is available for 409 surgical resected cases. 48 cases had chemotherapy and 21 cases had chemoradiation therapy.

\#ASC: adenosquamous carcinoma.

\section{Results}

\section{Entotic cell in cell structures in PDAC}

We identified 539 entotic-CICs within 537 PDAC (516 conventional PDAC and 21 ASC) cases in the MSK IMPACT Clinical Sequencing Cohort. Entotic-CICs per ten HPFs ranged from 0 to 3.06 (median 0.12) per neoplasm (Fig. $1 \mathrm{~b}$ and c). Per our predefined criteria (see Materials and Methods), 70 of 537 carcinomas (13.0\%) correspond to entotic-CIC positive PDACs. All $(70 / 70,100 \%)$ entoticCIC positive cases had two or more entotic-CICs and more than $80 \%$ (59/70 cases, $84.3 \%$ ) of cases had three or more. Representative CIC patterns ( $\sim 50$ CICs in 15 cases) were confirmed by E-Cadherin IHC that demonstrated membranous labeling of both the winner cell and the cell being engulfed (Fig. 1c).

\section{Clinical characteristic of entotic-CIC positive PDAC}

Entotic-CIC positive status was unrelated to age, gender in univariate analysis of the IMPACT cohort (Table 1). No significant differences were seen for entotic CIC positivity between PDAC and ASC in total cohort (Table 1), although when the analysis was limited to primary tumors only poorly differentiated adenocarcinoma (23 out of 111 samples, $20.7 \%)$ or ASC $(5 / 19,26.3 \%)$ had more entotic-CIC positivity than well $(2 / 75,2.7 \%)$ or moderately $(10 / 210$, $4.8 \%)$ differentiated adenocarcinoma tumors $(P<0.0001$, the two-sided Fisher's exact test) (Table 2). For primary 
surgical resected cases, cases with neoadjuvant chemo- or chemoradiation-therapy had less entotic-CIC (3/69, 4.3\%) compared with treatment naïve primary tumors (37/340, $10.9 \%)$, but this difference was not statistically significant ( $P=0.120$, the two-sided Fisher's exact test) (Table 2). No differences were confirmed between lymph node positive and negative cases (Table 2). Entotic-CIC positivity was significantly more prevalent in metastases (30 out of 122 samples, $24.6 \%)$ compared with unmatched primary tumors $(40 / 415$ samples, $9.6 \%)(P<0.0001$, the two-sided Fisher's exact test) (Table 1). Among metastatic sites, entotic CIC positive samples most often corresponded to liver metastases $(24 / 69,34.8 \%)$ (Table 3).

We next reviewed matched primary and liver metastasis samples from an independent cohort of patients who

Table 3 Metastatic characteristic of entotic-CIC positive PDAC.

\begin{tabular}{llrll}
\hline Metastasis location & \multicolumn{3}{c}{ Entotic-CIC } & \\
\cline { 3 - 4 } & Total & Positive & Negative & \% Positive \\
\hline Liver & 69 & 24 & 45 & $34.8 \%$ \\
Peritoneal cavity & 25 & 2 & 23 & $8.0 \%$ \\
Lung & 12 & 2 & 10 & $16.7 \%$ \\
Other $^{\text {a }}$ & 16 & 2 & 14 & $12.5 \%$ \\
\hline
\end{tabular}

athers include metastases of lymph nodes (6), soft tissue (2), brain (1), pleura (1), retroperitoneum (1), arm (1), skin (1), umbilicus (1), unknown (2). underwent an autopsy. This confirmed that liver metastases had more entotic-CICs than primary carcinomas $(P=0.023$, the Wilcoxon matched-pair signed rank test, two-sided) (Fig. 2a, b). To determine the extent that different sites of metastasis in the same patient exhibit entotic-CICs we further assessed available lung or peritoneal metastases in these patients. This revealed heterogeneity across the primary tumor and metastases in that entotic-CICs in one site were not indicative of a high number of entotic-CICs in another metastatic site in the same patient, though the degree of heterogeneity varied among cases (Fig. 2a, c).

\section{Genetic characteristics of entotic-CIC positive PDAC}

Entotic-CIC positive PDACs had a higher prevalence of TP53 mutations ( $P=0.041$, the Fisher's exact test, two-sided) and KRAS and MYC amplification $(P=0.025$ and 0.012 , the Fisher's exact test, two-sided) (Table 4). Deep deletions for CDKN2A, TP53, or SMAD4 were not statistically associated with entotic-CIC positivity (Table 4). To determine if a specific type of TP53 alteration was associated with the formation of entotic-CICs we compared lollipop plots of the mutational spectrum of TP53 in entotic-CIC positive vs. negative cases (Fig. 3). We found no difference between the prevalence of hotspot mutations $(P=0.331$, the Fisher's exact test, two-sided) nor did we find a difference in the proportion of truncating and non-truncating TP53 mutations between the two groups $(P=1.000$, the Fisher's exact test, two-sided).
Fig. 2 Heterogeneity of entotic-CICs in PDAC. a Entotic-CIC (per ten HPFs) in matched primary, and liver, peritoneal cavity and lung metastasis in end-stage PDAC autopsy cohort. b Representative histomorphologic images of entotic-CICs in primary and live and peritoneal cavity metastases in autopsy series MPAM06. More than one entotic-CICs were found in one HPF in liver metastasis. c Heterogeneity of entotic-CIC among metastatic organs in end-stage PDAC autopsy cohort. a

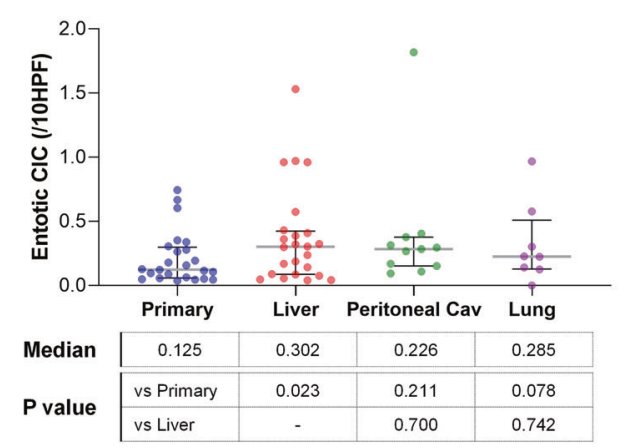

C

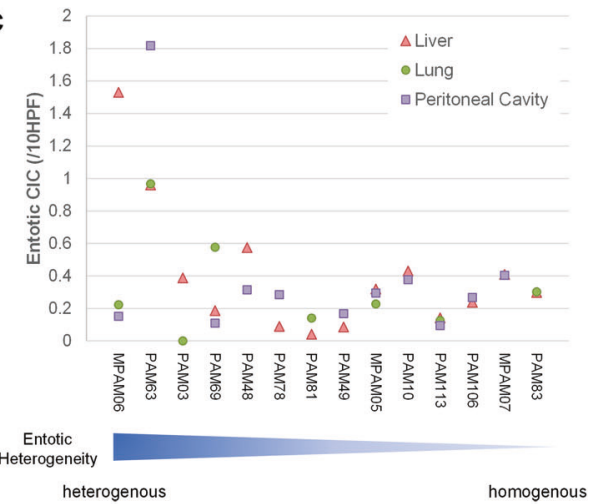

b

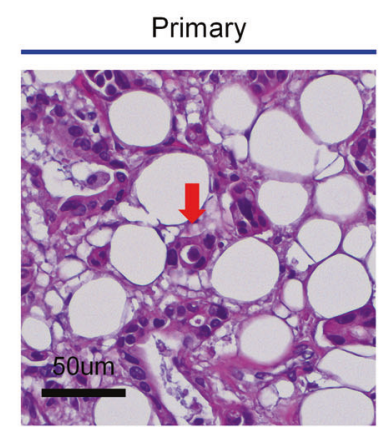

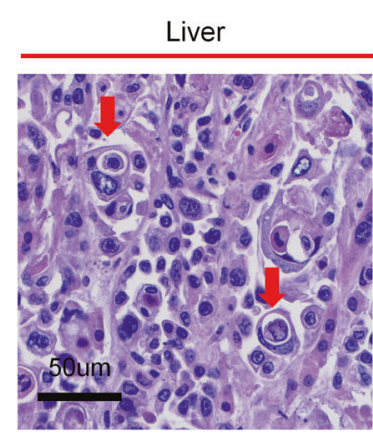

Peritoneal Cavity

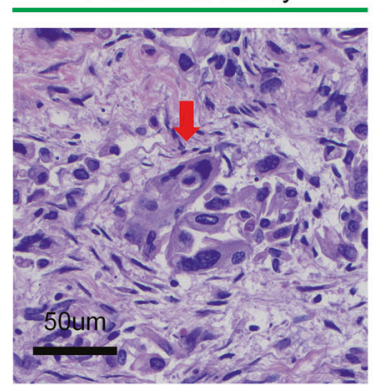


Table 4 Genetic characteristics of entotic-CIC positive PDAC.

\begin{tabular}{|c|c|c|c|c|c|c|c|}
\hline \multirow[t]{2}{*}{ Category } & \multirow[t]{2}{*}{ Gene } & & \multirow[b]{2}{*}{ Total } & \multicolumn{3}{|c|}{ Entotic-CIC } & \multirow[b]{2}{*}{$P$ Value } \\
\hline & & & & Positive & Negative & $\%$ Positive & \\
\hline \multirow[t]{20}{*}{ Mutation } & $K R A S$ & Mutated & 492 & 68 & 424 & $13.8 \%$ & 0.102 \\
\hline & & WT & 45 & 2 & 43 & $4.4 \%$ & \\
\hline & TP53 & Mutated & 399 & 59 & 340 & $14.8 \%$ & 0.041 \\
\hline & & WT & 138 & 11 & 127 & $8.0 \%$ & \\
\hline & $C D K N 2 A$ & Mutated & 137 & 24 & 113 & $17.5 \%$ & 0.078 \\
\hline & & WT & 400 & 46 & 354 & $11.5 \%$ & \\
\hline & SMAD4 & Mutated & 113 & 20 & 93 & $17.7 \%$ & 0.115 \\
\hline & & WT & 424 & 50 & 374 & $11.8 \%$ & \\
\hline & CDKN2AP16INK4A & Mutated & 97 & 15 & 82 & $15.5 \%$ & 0.410 \\
\hline & & WT & 440 & 55 & 385 & $12.5 \%$ & \\
\hline & ARIDIA & Mutated & 56 & 10 & 46 & $17.9 \%$ & 0.292 \\
\hline & & WT & 481 & 60 & 421 & $12.5 \%$ & \\
\hline & $C D K N 2 A P 14 A R F$ & Mutated & 59 & 10 & 49 & $16.9 \%$ & 0.313 \\
\hline & & WT & 478 & 60 & 418 & $12.6 \%$ & \\
\hline & RNF43 & Mutated & 29 & 4 & 25 & $13.8 \%$ & 0.782 \\
\hline & & WT & 508 & 66 & 442 & $13.0 \%$ & \\
\hline & KDM6A & Mutated & 28 & 5 & 23 & $17.9 \%$ & 0.393 \\
\hline & & WT & 509 & 65 & 444 & $12.8 \%$ & \\
\hline & $A P C$ & Mutated & 25 & 2 & 23 & $8.0 \%$ & 0.759 \\
\hline & & WT & 512 & 68 & 444 & $13.3 \%$ & \\
\hline \multirow[t]{20}{*}{$\mathrm{CNV}$} & $M Y C$ & Amplified & 14 & 5 & 9 & $35.7 \%$ & 0.025 \\
\hline & & No amplified & 523 & 65 & 458 & $12.4 \%$ & \\
\hline & $A K T 2$ & Amplified & 18 & 6 & 12 & $33.3 \%$ & 0.205 \\
\hline & & No amplified & 519 & 64 & 455 & $12.3 \%$ & \\
\hline & CCNE1 & Amplified & 10 & 3 & 7 & $30.0 \%$ & 0.130 \\
\hline & & No amplified & 527 & 67 & 460 & $12.7 \%$ & \\
\hline & KRAS & Amplified & 8 & 4 & 4 & $50.0 \%$ & 0.012 \\
\hline & & No amplified & 529 & 66 & 463 & $12.5 \%$ & \\
\hline & FGFR1 & Amplified & 6 & 1 & 5 & $16.7 \%$ & 0.569 \\
\hline & & No amplified & 531 & 69 & 462 & $13.0 \%$ & \\
\hline & $C D K N 2 A$ & Deep deletion & 45 & 6 & 39 & $13.3 \%$ & 1.000 \\
\hline & & No deletion & 492 & 64 & 428 & $13.0 \%$ & \\
\hline & CDKN2AP16INK4A & Deep deletion & 44 & 6 & 38 & $13.6 \%$ & 0.818 \\
\hline & & No deletion & 493 & 64 & 429 & $13.0 \%$ & \\
\hline & CDKN2AP14ARF & Deep deletion & 42 & 6 & 36 & $14.3 \%$ & 0.811 \\
\hline & & No deletion & 495 & 64 & 431 & $12.9 \%$ & \\
\hline & $C D K N 2 B$ & Deep deletion & 40 & 4 & 36 & $10.0 \%$ & 0.806 \\
\hline & & No deletion & 497 & 66 & 431 & $13.3 \%$ & \\
\hline & SMAD4 & Deep deletion & 18 & 2 & 16 & $11.1 \%$ & 1.000 \\
\hline & & No deletion & 519 & 68 & 451 & $13.1 \%$ & \\
\hline
\end{tabular}

The bold value means statistically significant $P$ value.

Each $P$ value was calculated with the Fisher's exact test, two-sided.
(Supplementary Information 1). The distribution of TP53 mutations also did not differ among CIC positive and negative carcinomas among primary and metastatic sites specifically (Supplementary Information 2). While a prior report suggested expression of KRAS-G12V in eating cells is associated with entosis [6], we observed no relationship of a specific KRAS hotspot mutation in our cohort $(P=0.501$, the Fisher's exact test, two-sided) (Supplementary Information 3). 

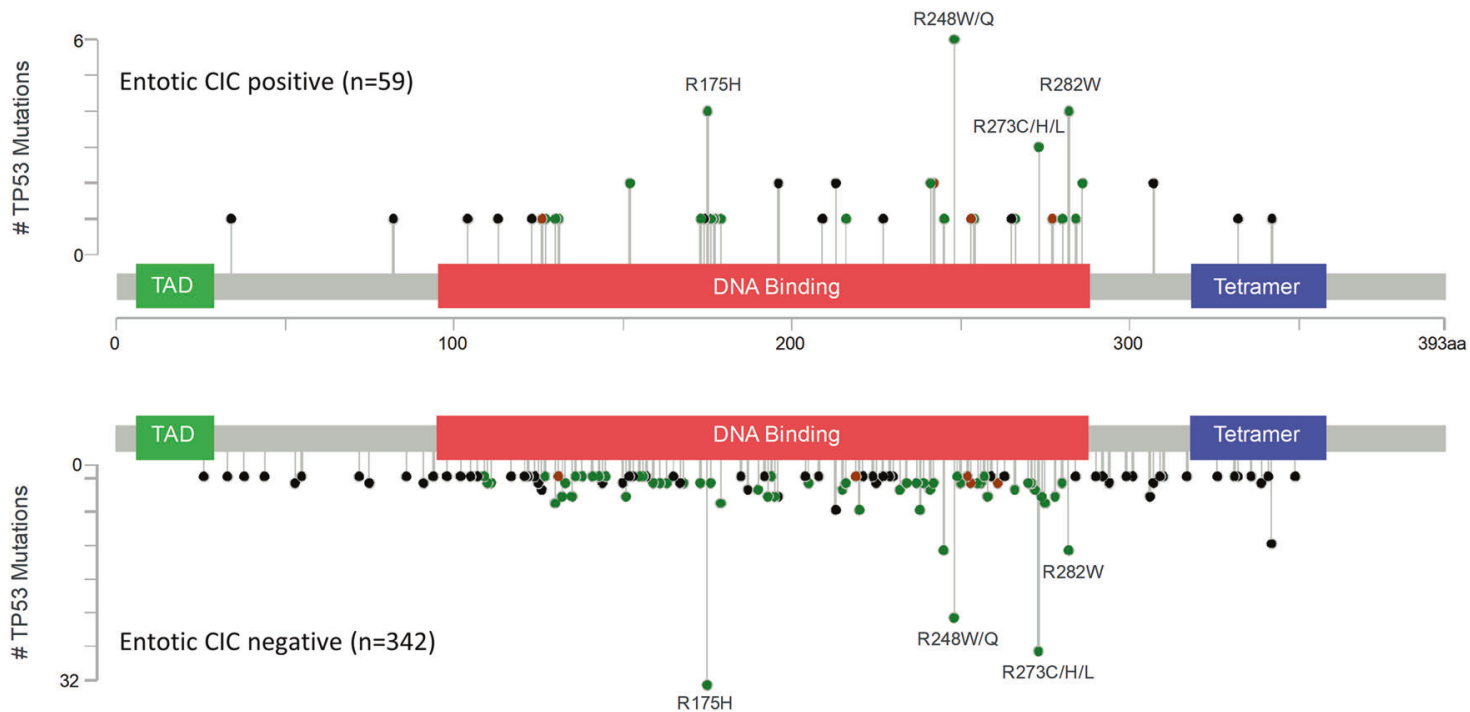

Fig. 3 Lolliplot of TP53 in entotic-CIC positive and negative PDACs. Number of total cases is shown in parentheses.

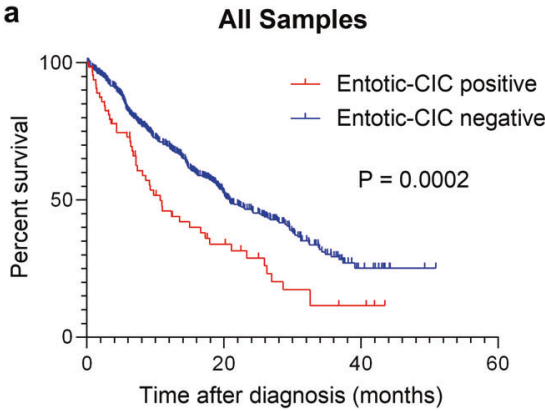

Fig. 4 Kaplan-Meier analysis of entotic-CIC positive and negative PDAC. a Entotic-CIC positive PDAC $(n=65)$ showed poorer prognosis than negative PDACs $(n=434)(P=0.0002$, the log-rank

\section{Outcome of entotic-CIC positive PDAC}

Kaplan-Meier analysis revealed significant poorer prognosis of entotic-CIC Positive PDAC when considering all cases $(P=0.0002$, the log-rank test) (Fig. 4a) or primary carcinomas only ( $P=0.021$, the log-rank test) (Fig. $4 b)$. Cox proportional hazards analysis showed positive entoticCICs, gender, tumor location, and KRAS amplification were all associated with a poor prognosis by both univariate and multivariate analyses (Tables 5 and 6). Entotic-CIC positive status was an independent prognostic variable by both univariate $(P<0.001$, Hazard ratio: 1.813) (Table 5) and multivariate analysis $(P=0.014$, Hazard ratio: 1.527) (Table 6). This significance identified by multivariate analysis was also confirmed when we use primary carcinomas only $(P=0.018$, Hazard ratio: 1.668) (Table 7).

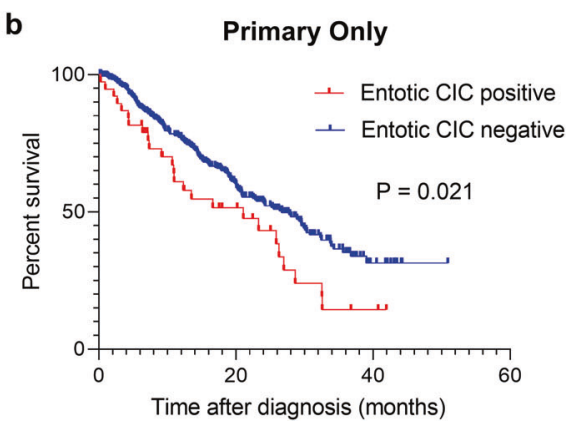

(Mantel-Cox) test) in all samples. b Entotic-CIC positive primary PDAC $(n=39)$ showed poorer prognosis than negative primary PDACs $(n=350)(P=0.021$, the log-rank (Mantel-Cox) test).

\section{Discussion}

We find that entosis in PDAC is a correlate of aggressive biology and an independent prognostic factor by multivariate analysis. Moreover, metastases appear to have a greater number of entotic-CICs than primary tumors. These findings not only validate prior reports in rectal, head, and neck and breast cancer and but provide a deeper understanding of the clinicopathologic relevance of entosis in PDAC.

Our finding that entotic-CICs are more prevalent in poorly differentiated PDAC parallels prior observations in breast cancer [9]. We extend this observation by showing that pancreatic ASC also have a high number of entoticCICs. This is consistent with our prior reports that squamous features in PDAC arise in the context of clonal 
Table 5 Entotic-CIC in PDAC and patient outcome (univariate analysis).

\begin{tabular}{|c|c|c|c|c|}
\hline \multicolumn{3}{|l|}{ Factor } & \multirow{2}{*}{$\begin{array}{l}P \text { value } \\
<\mathbf{0 . 0 0 1}\end{array}$} & \multirow{2}{*}{$\begin{array}{l}\text { Hazard ratio }(95 \% \text { confidence } \\
\text { interval) }\end{array}$} \\
\hline Clinicopathologic & Entotic-CIC & (Positive vs. negative) & & \\
\hline & Age & $(>65$ vs. $<=65)$ & 0.853 & $1.024(0.8-1.31)$ \\
\hline & Gender & (Male vs. female) & 0.010 & $1.380(1.082-1.761)$ \\
\hline & Location & $\begin{array}{l}\text { (Primary vs. } \\
\text { metastasis) }\end{array}$ & $<0.001$ & $0.327(0-0.423)$ \\
\hline & Histology & (PDA vs. ASQ) & 0.415 & $0.778(0.425-1.424)$ \\
\hline \multirow[t]{10}{*}{ Genetic } & $K R A S$ & (Mutated vs. WT) & 0.969 & $0.991(0.646-1.521)$ \\
\hline & TP53 & (Mutated vs. WT) & 0.173 & $1.216(0.918-1.611)$ \\
\hline & $C D K N 2 A$ & (Mutated vs. WT) & 0.094 & $1.259(0.962-1.649)$ \\
\hline & SMAD4 & (Mutated vs. WT) & 0.976 & $0.996(0.746-1.329)$ \\
\hline & CDKN2AP16INK4A & (Mutated vs. WT) & 0.384 & $1.141(0.848-1.534)$ \\
\hline & ARID1A & (Mutated vs. WT) & 0.395 & $1.179(0.807-1.725)$ \\
\hline & $C D K N 2 A P 14 A R F$ & (Mutated vs. WT) & 0.310 & $1.199(0.844-1.703)$ \\
\hline & RNF43 & (Mutated vs. WT) & 0.382 & $0.772(0.433-1.378)$ \\
\hline & $K D M 6 A$ & (Mutated vs. WT) & 0.828 & $1.062(0.619-1.82)$ \\
\hline & $A P C$ & (Mutated vs. WT) & 0.166 & $1.464(0.854-2.51)$ \\
\hline \multirow[t]{10}{*}{ CNA alteration } & $M Y C$ & $\begin{array}{l}\text { (Amplified vs. not } \\
\text { amplified) }\end{array}$ & 0.883 & $0.941(0.419-2.115)$ \\
\hline & $A K T 2$ & $\begin{array}{l}\text { (Amplified vs. not } \\
\text { amplified) }\end{array}$ & 0.399 & $1.297(0.709-2.371)$ \\
\hline & CCNE1 & $\begin{array}{l}\text { (Amplified vs. not } \\
\text { amplified) }\end{array}$ & 0.503 & $0.678(0.217-2.116)$ \\
\hline & $K R A S$ & $\begin{array}{l}\text { (Amplified vs. not } \\
\text { amplified) }\end{array}$ & $<0.001$ & $5.480(2.419-12.416)$ \\
\hline & FGFR1 & $\begin{array}{l}\text { (Amplified vs. not } \\
\text { amplified) }\end{array}$ & 0.117 & $2.490(0.796-7.788)$ \\
\hline & $C D K N 2 A$ & $\begin{array}{l}\text { (Deep deletion vs. no } \\
\text { deletion) }\end{array}$ & 0.010 & $1.631(1.122-2.372)$ \\
\hline & $C D K N 2 A P 16 I N K 4 A$ & $\begin{array}{l}\text { (Deep deletion vs. no } \\
\text { deletion) }\end{array}$ & 0.019 & $1.575(1.077-2.303)$ \\
\hline & $C D K N 2 A P 14 A R F$ & $\begin{array}{l}\text { (Deep deletion vs. no } \\
\text { deletion) }\end{array}$ & 0.016 & $1.604(1.091-2.358)$ \\
\hline & $C D K N 2 B$ & $\begin{array}{l}\text { (Deep deletion vs. no } \\
\text { deletion) }\end{array}$ & 0.216 & $1.291(0.862-1.934)$ \\
\hline & SMAD4 & $\begin{array}{l}\text { (Deep deletion vs. no } \\
\text { deletion) }\end{array}$ & 0.242 & $0.639(0.302-1.353)$ \\
\hline
\end{tabular}

The bold value means statistically significant $P$ value. evolution and that ASC has more entotic-CICs than poorly differentiated carcinoma [10]. Furthermore, our finding of an association of TP53 mutations with entotic-CIC parallels prior reports in lung cancer [5]. However, we now clarify the lack of an association with TP53 hotspot mutations. While the mechanisms by which TP53 alterations contribute to entosis is unknown, one putative molecular mechanism is that loss of TP53 or delta-133TP53 expression increases extracellular ATP release and the consequent activation of purinergic $\mathrm{P} 2 \mathrm{Y} 2$ receptors, which induces cell engulfment [15]. Alternatively, TP53 activation may cause upregulation of Rnd3, which inhibits ROCK1 or RhoA activities directly or indirectly, leading to polarized activation of RhoAactomyosin, which drives cell internalization to form CIC structures [16].

The reason for an increased prevalence of entotic-CICs in metastatic PDAC, specifically liver metastases, also remains to be determined. However, we expect that the mechanisms are multifactorial. For example, a recent report showed TP53 inactivation and KRAS amplification are more frequent in metastasis [17]. Our data confirms this phenomenon because of eight KRAS amplified samples, six were liver metastases and only two were primary carcinomas $(P<0.0001$, the chi square test with Yates correction). 
Table 6 Multivariate analysis of entotic-CIC in PDAC and patient.

\begin{tabular}{llll}
\hline Factor & & $P$ value & $\begin{array}{l}\text { Hazard ratio } \\
(95 \% \text { CI })\end{array}$ \\
\hline Entotic-CIC & (Present vs. absent) & $\mathbf{0 . 0 1 4}$ & $1.527(1.090-2.138)$ \\
Gender & (Male vs. female) & $\mathbf{0 . 0 0 5}$ & $1.431(1.115-1.836)$ \\
Location & (Primary vs. & $<\mathbf{0 . 0 0 1}$ & $0.360(0-0.474)$ \\
& metastasis) & & \\
TP53 & (Mutated vs. WT) & 0.328 & $1.153(0.866-1.535)$ \\
CDKN2A & (Mutated vs. WT) & 0.217 & $1.197(0.900-1.592)$ \\
APC & (Mutated vs. WT) & 0.409 & $1.184(0.792-1.771)$ \\
KRAS & (Amplified vs. not & $\mathbf{0 . 0 2 0}$ & $2.742(1.173-6.408)$ \\
FGFR1 & amplified) & & \\
& (Amplified vs. not & 0.944 & $0.959(0.295-3.119)$ \\
CDKN2A & (Deep deletion vs. no & 0.245 & $1.382(0.801-2.386)$ \\
& deletion) & & \\
\hline
\end{tabular}

Table 7 Multivariate analysis of entotic-CIC in PDAC (Primary Only) and patient.

\begin{tabular}{llll}
\hline Factor & & $P$ value & $\begin{array}{l}\text { Hazard ratio } \\
(95 \% \mathrm{CI})\end{array}$ \\
\hline Enototic-CIC & (Present vs. absent) & $\mathbf{0 . 0 1 8}$ & $1.668(1.092-2.548)$ \\
Gender & (Male vs. female) & 0.181 & $1.225(0.910-1.651)$ \\
TP53 & (Mutated vs. WT) & 0.060 & $1.435(0.986-2.090)$ \\
CDKN2A & (Mutated vs. WT) & 0.254 & $1.217(0.868-1.708)$ \\
APC & (Mutated vs. WT) & 0.106 & $1.825(0.881-3.781)$ \\
KRAS & (Amplified vs. not & $\mathbf{0 . 0 1 0}$ & 8.242 \\
& amplified) & & $(1.658-40.974)$ \\
FGFR1 & (Amplified vs. not & 0.384 & 2.420 \\
& amplified) & & $(0.331-17.674)$ \\
CDKN2A & (Deep deletion vs. no & 0.415 & $0.711(0.314-1.613)$ \\
& deletion) & & \\
\hline
\end{tabular}

While PDAC metastases does not appear to have a genetic basis [18], subclonal evolution at the genetic level with respect to gene dosages may select for the entotic phenotype. We suspect the tumor microenvironment may also play a role. For example, while primary tumors are rich in stromal cells and poorly vascularized, the liver is by contrast a highly vascular tissue with little stromal response until the metastases exceeds a critical mass of cells [19]. Thus, it is conceivable that changes in the microenvironment and available nutrients may select for the entotic phenotype within specific genetic contexts.

Poor prognosis in entotic-CIC high tumors that we report here have been previously reported in lung adenocarcinoma [5], HNSCC [7, 8], rectal and anal carcinoma [8]. However, given that only in HNSCC has entotic-CIC been shown to be an independent prognostic factor in HNSCC [7], this is the first demonstration of entotic -IC as an independent prognostic factor in adenocarcinoma. While we cannot totally exclude the possibility of simple dimensional overlap or emperipolesis [20] in tumor cells reported previously, we believe that our simple methods to evaluate entotic-CIC can be applied to practical diagnostic pathology. We also expect that our findings will also have implications for understanding entotic-CIC in other solid tumor types.

Acknowledgements We are grateful for Laura D. Wood and Ralph $\mathrm{H}$. Hruban for sample collection and helpful discussions. We gratefully acknowledge the members of the Molecular Diagnostics Service in the Department of Pathology for MSK IMPACT.

\section{Compliance with ethical standards}

Conflict of interest CAI-D receives research support from Bristol Myers Squibb. DSK is a consultant and equity holder to Paige.AI, consultant to Merck Pharmaceuticals, and receives royalties from UpToDate and the American Registry of Pathology. EMO'R has research funding to MSK from Celgene/BMS, BioNTech, ActaBiologica, AstraZenica, Silenseed, Arcus and Gossamer and is a consult to Merck, Cytomx, BioLineRx, Targovax, Celgene/BMS and Loxo, Polaris and Rafael. All other authors declare no competing interests.

Publisher's note Springer Nature remains neutral with regard to jurisdictional claims in published maps and institutional affiliations.

Open Access This article is licensed under a Creative Commons Attribution 4.0 International License, which permits use, sharing, adaptation, distribution and reproduction in any medium or format, as long as you give appropriate credit to the original author(s) and the source, provide a link to the Creative Commons license, and indicate if changes were made. The images or other third party material in this article are included in the article's Creative Commons license, unless indicated otherwise in a credit line to the material. If material is not included in the article's Creative Commons license and your intended use is not permitted by statutory regulation or exceeds the permitted use, you will need to obtain permission directly from the copyright holder. To view a copy of this license, visit http://creativecommons. org/licenses/by/4.0/.

\section{References}

1. Galluzzi L, Vitale I, Aaronson SA, Abrams JM, Adam D, Agostinis P, et al. Molecular mechanisms of cell death: recommendations of the Nomenclature Committee on Cell Death 2018. Cell Death Differ. 2018;25:486-541.

2. Overholtzer M, Mailleux AA, Mouneimne G, Normand G, Schnitt SJ, King RW, et al. A nonapoptotic cell death process, entosis, that occurs by cell-in-cell invasion. Cell. 2007;131:966-79.

3. Riegman M, Bradbury MS, Overholtzer M. Population dynamics in cell death: mechanisms of propagation. Trends Cancer 2019;5:558-68.

4. Hamann JC, Surcel A, Chen R, Teragawa C, Albeck JG, Robinson DN, et al. Entosis is induced by glucose starvation. Cell Rep. 2017;20:201-10.

5. Mackay HL, Moore D, Hall C, Birkbak NJ, Jamal-Hanjani M, Karim SA, et al. Genomic instability in mutant p53 cancer cells upon entotic engulfment. Nat Commun. 2018;9:3070. 
6. Sun Q, Luo T, Ren Y, Florey O, Shirasawa S, Sasazuki T, et al. Competition between human cells by entosis. Cell Res. 2014;24:1299-310.

7. Schenker H, Buttner-Herold M, Fietkau R, Distel LV. Cell-in-cell structures are more potent predictors of outcome than senescence or apoptosis in head and neck squamous cell carcinomas. Radiat Oncol. 2017;12:21.

8. Schwegler M, Wirsing AM, Schenker HM, Ott L, Ries JM, Büttner-Herold M, et al. Prognostic value of homotypic cell internalization by nonprofessional phagocytic cancer cells. Biomed Res Int. 2015;2015:359392.

9. Krajcovic M, Johnson NB, Sun Q, Normand G, Hoover N, Yao E, et al. A non-genetic route to aneuploidy in human cancers. Nat Cell Biol. 2011;13:324-30.

10. Hayashi A, Fan J, Chen R, Ho Y, Makohon-Moore AP, Lecomte $\mathrm{N}$, et al. A unifying paradigm for transcriptional heterogeneity and squamous features in pancreatic ductal adenocarcinoma. Nat Cancer 2020;1:59-74.

11. Gao J, Aksoy BA, Dogrusoz U, Dresdner G, Gross B, Sumer SO, et al. Integrative analysis of complex cancer genomics and clinical profiles using the cBioPortal. Sci Signal 2013;6:1.

12. Cheng DT, Mitchell TN, Zehir A, Shah RH, Benayed R, Syed A, et al. Memorial sloan kettering-integrated mutation profiling of actionable cancer targets (MSK-IMPACT): a hybridization capture-based next-generation sequencing clinical assay for solid tumor molecular oncology. J Mol Diagn. 2015;17:251-64.

13. WHO Classification of Tumours Editorial Board. Digestive System Tumours, WHO Classification of Tumours, 5th Edition. IARC publications, Lyon, 2019.

14. Sun Q, Cibas ES, Huang H, Hodgson L, Overholtzer M. Induction of entosis by epithelial cadherin expression. Cell Res. 2014;24:1288-98.

15. Syed Qasim Raza. Caractérisation des bases moléculaires de l'entose (Article in French). 2012.

16. Liang J, Niu Z, Yu X, Zhang B, Wang M, Ruan B, et al. Counteracting Genome Instability by p53-dependent Mintosis. bioRxiv. 2020. 2020.01.16.908954.

17. Connor AA, Denroche RE, Jang GH, Lemire M, Zhang A, ChanSeng-Yue $M$, et al. Integration of genomic and transcriptional features in pancreatic cancer reveals increased cell cycle progression in metastases. Cancer Cell 2019;35:267-82.

18. Makohon-Moore AP, Zhang M, Reiter JG, Bozic I, Allen B, Kundu D, et al. Limited heterogeneity of known driver gene mutations among the metastases of individual patients with pancreatic cancer. Nat Genet. 2017;49:358-66.

19. Yuan S, Norgard RJ, Stanger BZ. Cellular plasticity in cancer. Cancer Discov. 2019;9:837-51.

20. Xia P, Wang S, Guo Z, Yao X. Emperipolesis, entosis and beyond: dance with fate. Cell Res. 2008;18:705-7. 